\title{
Prognostic Role of Prothrombin Time Activity, Prothrombin Time, Albumin/Globulin Ratio, Platelets, Sex, and Fibrinogen in Predicting Recurrence-Free Survival Time of Renal Cancer
}

This article was published in the following Dove Press journal:

Cancer Management and Research

\author{
Zichen Bian ${ }^{1, *}$ \\ Jialin Meng ${ }^{1} *$ \\ Qingsong Niu' \\ Xiaoyan Jin ${ }^{2}$ \\ Jinian Wang ${ }^{3}$ \\ Xingliang Feng' \\ Hong Che ${ }^{4}$ \\ Jun Zhou' \\ Li Zhang' \\ Meng Zhang ${ }^{1,5}$ \\ Chaozhao Liang' \\ 'Department of Urology, The First \\ Affiliated Hospital of Anhui Medical \\ University; Anhui Province Key \\ Laboratory of Genitourinary Diseases, \\ Anhui Medical University; The Institute of \\ Urology, Anhui Medical University, Hefei, \\ People's Republic of China; ${ }^{2}$ The Second \\ Clinical College of Anhui Medical \\ University, Hefei, Anhui, People's \\ Republic of China; ${ }^{3}$ Clinical Skills Training \\ Center, The First Affiliated Hospital of \\ Anhui Medical University, Hefei 230022, \\ Anhui, People's Republic of China; \\ ${ }^{4}$ Department of Cardiac Surgery, The \\ First Affiliated Hospital of Anhui Medical \\ University, Hefei, People's Republic of \\ China; ${ }^{5}$ Institute of Urology of Shenzhen \\ University, The Third Affiliated Hospital \\ of Shenzhen University, Shenzhen Luohu \\ Hospital Group, Shenzhen 518000, \\ People's Republic of China
}

*These authors contributed equally to this work

Correspondence: Chaozhao Liang; Meng Zhang

Email liang_chaozhao@ahmu.edu.cn; zhangmeng1930@I26.com
Background: To help with the clinical practice of renal cancer patients, prognostic models are urgently warranted. We hunted and identified prognostic variables associated with recurrence-free survival (RFS) for renal cancer patients.

Patients and Methods: In this retrospective study, 187 renal cancer patients who had received curative radical/partial nephrectomy between November 2011 and January 2017 were enrolled in the current study. These patients were randomly split into the training $(\mathrm{n}=$ $95)$ and validation sets $(n=92)$ by the ratio of $1: 1$. Univariate and multivariable Cox regression analyses were used to establish the nomogram, which was then evaluated by receiver operating characteristic (ROC) and Kaplan-Meier (K-M) analyses.

Results: Patient characteristics and outcomes were well balanced between the training and validation sets; the median RFS values were 54.1 months and 58.9 months for the training and validation cohorts, respectively. The final nomogram included six independent prognostic variables (prothrombin time (\%), prothrombin time (second), albumin/globulin ratio, platelets, sex and fibrinogen). The mean values of RFS for the low- and high-risk groups defined by a prognostic formula were $56.22 \pm 18.50$ months and $49.54 \pm 23.57$ months, respectively, in the training cohort and were $59.00 \pm 19.50$ months and $53.32 \pm 19.95$ months, respectively, in the validation cohort. The significance and stability of the model were tested by the time-dependent K-M model and ROC curves, respectively.

Conclusion: Our validated prognostic model incorporates variables routinely collected from renal cancer patients, identifying subsets of patients with different survival outcomes, which provides useful information for patient care and clinical trial design.

Keywords: renal cancer, recurrence, nomogram

\section{Introduction}

Renal cancer is the sixth most frequent cancer in men and the 10th in women around the world. ${ }^{1}$ Most renal cancers can be diagnosed at an early stage, but tumour-specific mortality has continuously increased in the past decades. Moreover, there are approximately $1 / 5$ to $3 / 10$ renal cancer patients who will step into the metastasis stage after the initial radical or partial nephrectomy. ${ }^{2}$ On the other hand, renal cancer is insensitive to radiotherapy and chemotherapy; therefore, the prognosis of renal cancer patients is unfavourable, and a great number of patients die from the disease. ${ }^{3}$ Thus, more effective biomarkers that could forecast disease progression are warranted. 
Currently, numerous publications have reported the prognosis predictive value of clinical parameters among different tumours, ${ }^{4-6}$ as well as renal cancer. ${ }^{7}$ The clinical parameters are usually used to evaluate the basic stability of the internal environment of the human body, and the abnormal results always indicated the unbalanced internal environment that was associated with several diseases. Globulin (GLO) is one of the significant components of serum proteins and always responds to chronic inflammation with an increase in serum levels. ${ }^{8}$ Elevated serum GLO plays an essential role in several diseases, ${ }^{9}$ such as cancer, chronic liver disease, rheumatoid diseases, diabetes mellitus, and nephrotic syndrome. Moreover, the counts of several blood cells or their ratios were also reported as prognostic biomarkers for cancer. Platelets are regarded as multifunctional cells and participate in the immune response, allergy, tissue regeneration, inflammation, and lymphangiogenesis processes. The platelet count (PLT) has been reported to be associated with the prognoses of pancreatic cancer, ${ }^{10}$ cervical cancer, ${ }^{11}$ and gallbladder cancer. ${ }^{12}$ Some studies have been done by categorizing the relationship between cancer and coagulation function. Cancer cells are able to activate the coagulation system, while haemostatic factors also play a role in tumour progression. ${ }^{13,14}$ For example, the high pretreatment fibrinogen and D-dimer levels are reportedly related to poor overall survival (OS) in endometrial cancer patients. ${ }^{15,16}$ Thus, the clinical haematological parameters-based prognostic signatures are easily obtained as the prognosis forecast tools for patients with tumours. ${ }^{7}$

In the current study, we focused on the establishment and validation of a haematological parameters-based prognostic signature for renal cancer patients. A total of 187 renal cancer patients were registered. The basic information, haematological parameters, and follow-up data were all recorded and analysed. We built a nomogram to help the patients be aware of the recurrence risk by themselves as well.

\section{Patients and Methods}

\section{Patients and Inclusion Criteria}

The retrospective study involved 187 patients with renal cancer who were treated at the Department of Urology, The First Affiliated Hospital of Anhui Medical University between November 2011 and January 2017. Patients with the completed laboratory results and demographic and pathological data were enrolled, as well as the acquirable follow-up information. The patients who lacked any of the above mentioned data were not suitable for the current study. The current study was approved by the
Institutional Review Board of the First Affiliated Hospital of Anhui Medical University (anyiyifuyuanlunshen-kuai-PJ-2019-09-11).

\section{Follow-Up Record}

The follow-up evaluations included clinical laboratory tests and radiological examinations. All patients were followed-up via telephone interviews. The last follow-up was completed on 1 November 2019. The observed endpoint of renal cancer patients was tumour recurrence determined by the result based on radiological examination. The other patients were set as censored or recurrence-free ones.

\section{Clinical and Laboratory Parameters}

All clinicopathological data, including laboratory data, tumour stage, and demographic settings, were retrieved from the electronic medical records at our hospital. The American Joint Committee on Cancer (AJCC) TNM staging system (8th edition) was executed to determine the tumour stage. The characterizations of the laboratory parameter were demonstrated as below:

Absolute neutrophil count (NEUT); absolute lymphocyte count (LYMPH); red blood cell count (RBC); haemoglobin (HGB); haematocrit value (HCT); platelet count (PLT); neutrophil-to-lymphocyte ratio (NLR); albumin (ALB); globulin (GLO); albumin to gamma-glutamyltransferase ratio (AGR); direct bilirubin (DBIL); indirect bilirubin (IBIL); alanine transaminase (ALT); aspartate aminotransferase (AST); blood urea nitrogen (BUN); creatinine (CRE); uric acid (UA); plasma prothrombin time (PT (sec)); "international normalized ratio (INR); plasma prothrombin time activity (PT-\%); activated partial thromboplastin time (APTT); thrombin time (TT (sec)); fibrinogen (FIB (g/l)); pathological stage-T (stage-T); pathological stage-N (stage-N); and the AJCC staging system. The classifications of these laboratory characteristics are demonstrated in Table S1.

\section{Statistical Analysis}

All the enrolled samples were 1:1 random sampling without replacement by using sample() $R$ package. The randomizing process was blind to the demographic information or laboratory test results, which can ensure that the training and validation cohorts are independent to clarify the key points we focused on. Univariate analysis was applied to determine the significance of variables concerning RFS. A multivariate Cox regression model was implemented to establish the model for the prediction of RFS. We used the Kaplan-Meier (K-M) method and the Log rank test to 
compare the patients' clinical endpoints. In addition, the time-dependent receiver operative characteristic (tROC) curve and time-dependent area under the curve (tAUC) were applied to determine the stability of the classifier. We used R (http://www.R-project.org) software to make all statistical analyses. A two-sided $P<0.05$ was considered statistically significant.

\section{Results}

\section{Clinicopathological Features}

Baseline demographics and disease characteristics were balanced between the training set $(n=95)$ and the validation set $(\mathrm{n}=92)$ (Figure 1$)$. Table 1 summarizes the patients' characteristics in both the training and validation cohorts. Overall, the age of the enrolled patients was 56.72 \pm 12.68 years old. Of them, $118(63.0 \%)$ were male and 69 $(37.0 \%)$ were female. In all, 154, 19, 12, and 2 patients were at stage I, II, III and IV, respectively. The median follow-up time from diagnosis was 54.609 months (range 1 month to 97.2 months). Forty patients were diagnosed with a recurrence during the follow-up period.

\section{Univariate Analysis Indicted the Potential Prognostic Factors}

We performed the univariate analysis to determine the RFSrelated candidates from the 29 elements. The results indicated that age $(\mathrm{HR}=4.537,95 \% \mathrm{CI}: 1.653-12.459$, $P<0.05)$, PT $(\mathrm{sec})(\mathrm{HR}=6.806,95 \% \mathrm{CI}: 1.972-23.483$, $P<0.05)$, PT-INR (HR $=8.572,95 \%$ CI: $1.942-37.844, P<$ 0.05), PLT (HR = 3.154, 95\% CI: 1.252-7.946, $P<0.05)$, stage $(\mathrm{HR}=3.795,95 \% \mathrm{CI}: 1.271-11.331, P<0.05)$, FIB $(\mathrm{g} /$ 1) $(\mathrm{HR}=2.160,95 \% \mathrm{CI}: 1.010-4.618, P<0.05)$ and stage-T $(\mathrm{HR}=3.795,95 \% \mathrm{CI}: 1.271-11.331, P<0.05)$ were the risk factors for recurrence, while the PT - $\%(\mathrm{HR}=0.115,95 \% \mathrm{CI}$ : $0.039-0.345, P<0.05)$, AGR (HR $=0.155,95 \%$ CI: $0.046-$ $0.525, P<0.05)$, HCT $(\mathrm{HR}=0.286,95 \% \mathrm{CI}: 0.125-0.652$, $P<0.05)$, LYMPH (HR $=0.246,95 \%$ CI: $0.090-0.677, P<$ 0.05), HGB (HR $=0.343,95 \%$ CI: $0.151-0.777, P<0.05)$, and sex $(\mathrm{HR}=0.250,95 \% \mathrm{CI}: 0.074-0.849, P<0.05)$ were the protective factors for recurrence (Table 2).

As mentioned above, the analysis by means of a univariate method identified thirteen prognostic instances related to unfavourable RFS. The subsequent multivariate analysis found that the PT (\%), PT (sec), AGR, PLT, sex and FIB (g/l) were still statistically significant (Table 3). Based on these findings, subsequent analyses were performed.

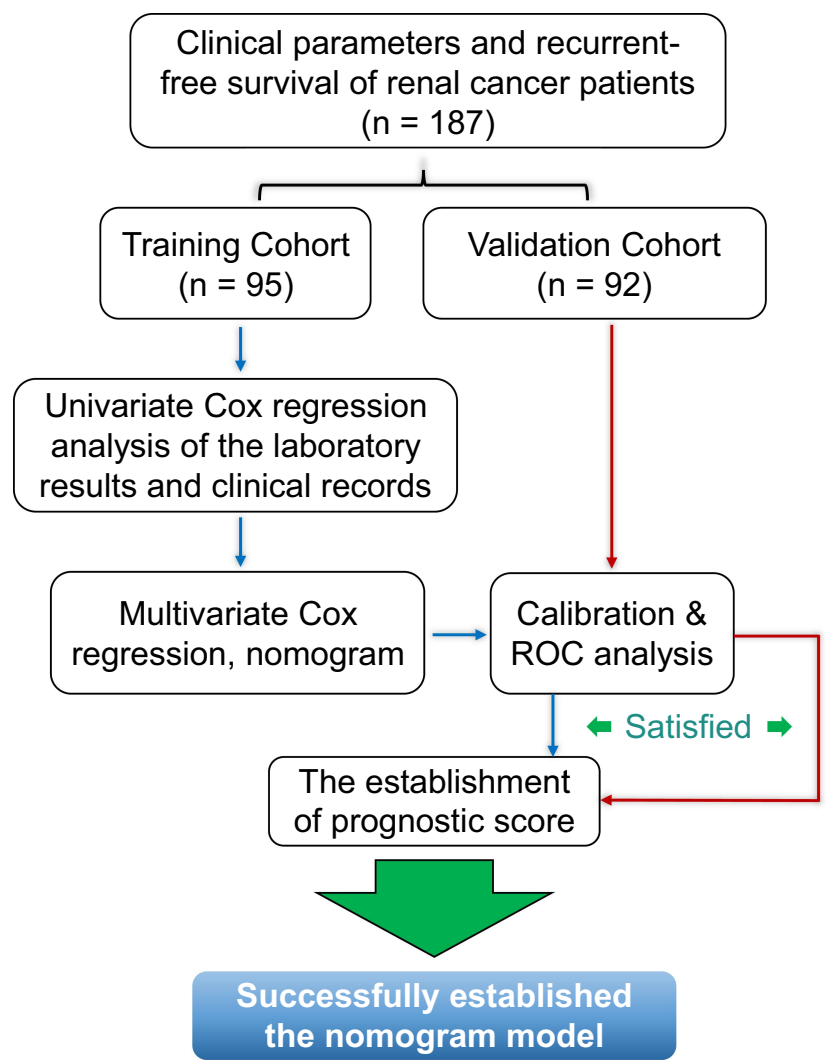

Figure I Flow chart showed the designation of current study.

\section{Prognostic Nomogram for the RFS of Renal Cancer Patients}

To integrate the data that contains all vital independent factors of RFS in the testing syngeneic group, a prognostic nomogram was made (Figure 2A). The C-index for the prediction of RFS was 0.810 in the training set. The prediction of the nomogram was optimally accorded with the actual observed value in the validation and training groups by calibration plot of the feasibility of 3- or 5-year survival after surgery (Figure 2B-E).

\section{ROC and K-M Analyses}

Concordance for predicting RFS as assessed by the tAUC scores for the four-variable model was $0.768,0.852$ and 0.790 at $1-$, 3- and 5-year RFS in the training set, respectively, while the tAUC scores were $0.683,0.756$, and 0.695 at 1-, 3- and 5-year RFS in the validation set, respectively (Figure 3). Although the multivariate analysis excluded the clinicopathological features, we performed the nomogram by combining the six variable-based classifier, age and tumour stage; the results suggested that our classifier showed the best predictive values compared to the tumour stage, and 
Table I Clinicopathological Features of the Enrolled Renal Cancer Patients

\begin{tabular}{|c|c|c|c|c|c|}
\hline Parameters & Training Cohort $(n=95)$ & Percent (\%) & Validation Cohort $(n=92)$ & Percent (\%) & P-value \\
\hline Age (mean \pm SD) & \multicolumn{2}{|l|}{$56.790 \pm 13.268$} & \multicolumn{2}{|l|}{$56.663 \pm 12.126$} & $0.946^{\dagger}$ \\
\hline Gender & & & & & $0.880^{\S}$ \\
\hline Male & 59 & $62.10 \%$ & 59 & $64.13 \%$ & \\
\hline Female & 36 & $37.90 \%$ & 33 & $35.87 \%$ & \\
\hline pT status & & & & & $0.588^{\S}$ \\
\hline $\mathrm{TI}+\mathrm{T} 2$ & 89 & $93.68 \%$ & 84 & $91.30 \%$ & \\
\hline $\mathrm{T} 3+\mathrm{T} 4$ & 6 & $6.32 \%$ & 8 & $8.70 \%$ & \\
\hline pN status & & & & & $0.117^{\S}$ \\
\hline No & 95 & $100 \%$ & 89 & $96.74 \%$ & \\
\hline $\mathrm{NI}$ & 0 & $0.00 \%$ & 3 & $3.26 \%$ & \\
\hline pM status & & & & & $1.000^{\S}$ \\
\hline MO & 95 & $100.00 \%$ & 92 & $100.00 \%$ & \\
\hline MI & 0 & $0.00 \%$ & 0 & $0.00 \%$ & \\
\hline pTNM stage & & & & & $0.588^{\S}$ \\
\hline$I+I I$ & 89 & $93.68 \%$ & 84 & $91.30 \%$ & \\
\hline III + IV & 6 & $6.32 \%$ & 8 & $8.70 \%$ & \\
\hline Age & & & & & $\left.0.77\right|^{\S}$ \\
\hline$\leq 55$ & 47 & $49.47 \%$ & 48 & $52.17 \%$ & \\
\hline$>55$ & 48 & $50.53 \%$ & 44 & $47.83 \%$ & \\
\hline
\end{tabular}

Notes: ${ }^{\dagger}$ Student $T$-Test; ${ }^{\S}$ Fisher Exact Probability Test.

Abbreviations: SD, standard deviation; ccRCC, clear cell renal cell carcinoma.

the combination of classifier, age and tumour stage showed better predictive values (Figure 4).

A risk score was determined by regression coefficients from the training set for the six variables. An algorithm was established accordingly. Patients who were in the training group were stratified as being at high- and low-risk of recurrence according to the score of median risk (Figure $5 \mathrm{~A}-\mathrm{C}$ ). The mean RFS among low-risk patients was $56.22 \pm 18.50$ months, whereas among high-risk patients, the mean RFS was $49.54 \pm 23.57$ months. In addition, our findings were further proved in an internal validation set. The patients who were validated were also stratified as being high- and lowrisk for recurrence (Figure 5D-F), which refers to the median risk score that came from the training group. The mean RFS among the low- and high-risk patients was $59.00 \pm 19.50$ months and $53.32 \pm 19.95$ months, respectively, in the validation cohort.

\section{Discussion}

The incidence of renal cancer has rapidly increased by approximately $2 \%$ worldwide during the last decades. ${ }^{17}$ Although advancements have been made in managing renal masses, long-term survival remains unsatisfactory; most patients with renal cancer still die of this disease. Therefore, renal cancer patients should receive close follow-up; at the same time, reliable prognostic biomarkers that evaluate postoperative risk and guide individualized treatment for renal cancer patients are equally necessary.

In recent years, numerous studies have investigated a wide variety of prognostic factors, such as TNM stage, ${ }^{17}$ Fuhrman's grade, and tumour size. ${ }^{18}$ However, these prognostic variables cannot always display the accurate predictions due to the limitation of significant tumour heterogeneity in renal cancer patients. ${ }^{19}$ Thus, novel biomarkers that can distinguish the high-risk renal cancer patients and improve clinical outcomes are desperately needed. In the past few years, nomograms have had a high development and been more precise than the traditional staging methods for predicting prognosis with regard to some cancers. ${ }^{20,21}$ Here, we performed univariate and multivariate Cox regression analyses and finally identified six RFS-related factors, including PT (\%), PT (sec), AGR, PLT, sex and FIB (g/l). Subsequently, we constructed a precise prognostic nomogram for renal cancer 
Table 2 Univariate Analysis Based on the Training Set

\begin{tabular}{|l|l|l|l|l|}
\hline Variables & HR & $\mathbf{9 5 \%}$ Low & 95\% High & P-value \\
\hline Sex & 0.250 & 0.074 & 0.849 & $0.026^{*}$ \\
Age & 4.538 & 1.653 & 12.459 & $0.003^{*}$ \\
BP & 0.845 & 0.330 & 2.167 & 0.727 \\
NEUT & 1.990 & 0.671 & 5.899 & 0.215 \\
LYMPH & 0.246 & 0.090 & 0.677 & $0.007^{*}$ \\
NLR & 2.197 & 0.859 & 5.619 & $0.10 I^{*}$ \\
RBC & 0.546 & 0.234 & 1.270 & 0.160 \\
HGB & 0.343 & 0.151 & 0.777 & $0.010^{*}$ \\
HCT & 0.286 & 0.125 & 0.652 & $0.003^{*}$ \\
PLT & 3.154 & 1.252 & 7.946 & $0.015^{*}$ \\
ALB & 0.492 & 0.200 & 1.208 & 0.121 \\
AGR & 0.155 & 0.046 & 0.525 & $0.003^{*}$ \\
GLO & 1.521 & 0.510 & 4.536 & 0.452 \\
DBIL & 1.352 & 0.464 & 3.941 & 0.580 \\
IBIL & 0.921 & 0.335 & 2.529 & 0.873 \\
ALT & 0.532 & 0.137 & 2.060 & 0.360 \\
AST & 0.704 & 0.262 & 1.891 & 0.486 \\
BUN & 1.317 & 0.533 & 3.257 & $0.55 \mathrm{I}$ \\
CRE & 0.978 & 0.408 & 2.344 & 0.960 \\
UA & 0.933 & 0.406 & 2.147 & 0.871 \\
PT (sec) & 6.806 & 1.972 & 23.483 & $0.002^{*}$ \\
PT-INR & 8.572 & 1.942 & 37.844 & $0.005^{*}$ \\
PT (\%) & 0.115 & 0.039 & 0.345 & $<0.001 *$ \\
APTT & 1.384 & 0.340 & 5.630 & 0.650 \\
FIB (g/l) & 2.160 & 1.010 & 4.618 & $0.047^{*}$ \\
TT (sec) & 0.806 & 0.016 & 41.853 & 0.915 \\
Pathological T & 3.795 & 1.271 & 11.331 & $0.017^{*}$ \\
Pathological N & NA & NA & NA & NA \\
Stage & 3.795 & 1.271 & 11.331 & $0.017^{*}$ \\
\hline Note: $<0.05$ & & & & \\
\hline
\end{tabular}

Note: $* \mathrm{P}<0.05$

Abbreviations: HR, hazard ratio; BP, blood pressure; NEUT, absolute neutrophil count; LYMPH, absolute lymphocyte count; NLR, neutrophil-to-lymphocyte ratio; RBC, red blood cell count; HGB, hemoglobin; HCT, hematocrit value; PLT, platelet count; ALB, albumin; AGR, albumin to gamma-glutamyltransferase ratio; GLO, globulin; DBIL, direct bilirubin; IBIL, indirect bilirubin; ALT, alanine transaminase; AST, aspartate aminotransferase; BUN, blood urea nitrogen; CRE, creatinine; UA, uric acid; PT (sec), plasma prothrombin time; PT-INR, international normalized ratio; PT (\%), plasma prothrombin time activity; APTT, activated partial thromboplastin time; FIB (g/l), fibrinogen; TT (sec), thrombin time.

patients. The nomogram played a good part in predicting the rate of survival, and the prediction gains support from the C-index ( 0.810 and 0.765 for the training and validation cohorts, respectively) and the calibration curve. The nomogram predicts RFS survival more accurately than the AJCC staging system. Although the multivariate analysis excluded tumour stage as an independent factor for the prediction of RFS, we performed an additional nomogram (nomogram2) by combining the six laboratory-based classifier, tumour stage and age. The results suggested nomogram2 had an increasing predictive value compared to these variables alone.
Table 3 Multivariable Analysis of Prognostic Variables in the Training Set

\begin{tabular}{|l|l|l|l|l|l|}
\hline Variables & $\begin{array}{l}\text { Co- } \\
\text { ef }\end{array}$ & $\begin{array}{l}\text { Exp (co- } \\
\text { ef) }\end{array}$ & $\begin{array}{l}\text { Se (co- } \\
\text { ef) }\end{array}$ & $\mathbf{z}$ & P value \\
\hline PT (\%) & 4.577 & 0.010 & 0.576 & 7.949 & $<0.00 I^{*}$ \\
(Normal) & & & & & \\
PT (sec) & 1.576 & 4.834 & 0.643 & $2.45 \mathrm{I}$ & $0.014^{*}$ \\
(Normal) & & & & & \\
PT (sec) (High) & 1.366 & 0.255 & 0.643 & 2.125 & $0.034^{*}$ \\
AGR (High) & 1.746 & 0.174 & 0.629 & 2.777 & $0.005^{*}$ \\
PLT (High) & 2.735 & 15.410 & 0.490 & $5.58 \mathrm{I}$ & $<0.00 I^{*}$ \\
Sex (Female) & 1.960 & 0.141 & 0.626 & 3.130 & $0.002^{*}$ \\
FIB (g/l) & 1.648 & 5.197 & 0.429 & 3.845 & $<0.00 I^{*}$ \\
(Normal) & & & & & \\
\hline
\end{tabular}

Note: $* \mathrm{P}<0.05$.

Abbreviations: Co-ef, co-efficient; Exp (co-ef), exponent of the coefficient; PT (\%), plasma prothrombin time activity; PT (sec), plasma prothrombin time; AGR, albumin to gamma-glutamyltransferase Ratio; PLT, platelet count; FIB (g/l), fibrinogen (gram/liter).

Notably, it has already been shown that the haemostatic activities induced by cancer could promote tumour metastasis, development, and progression. ${ }^{22-24}$ Actually, the connection between tumour spread, progression and the aberrant parameter of fibrinolytic haemostasis may reduce overall survival (OS) of patients with tumours. ${ }^{25}$ In the study by $\mathrm{Li}$ et al, ${ }^{26}$ they pointed out that the reduction in pretreatment thrombin time (TT) has something to do with the lessening of oesophageal carcinoma (ESCC) survival. Tas et $\mathrm{al}^{27}$ also expounded that the international normalized ratio (INR) and the prothrombin time (PT) have some prognostic values in lung cancer. In addition, some original studies indicated that hyperfibrinogenaemia was also considered an independent prognostic predictor of melanoma $^{28}$ and gallbladder cancer. ${ }^{29}$ Fibrinogen (FIB) is a kind of clotting factor ${ }^{30}$ and has been proven to have prognostic value in breast cancer. ${ }^{31}$ Here, we first reported that the PT (\%), PT (sec) and FIB could serve as independent risk factors for prediction of RFS.

As ALB and GLO are the two major serum proteins in the human body, low levels of ALB and high levels of GLO reflect malnutrition and a chronic inflammatory state. $^{32,33}$ Equally, the AGR can also reflect the nutritional and inflammatory status. Malnutrition influences the function of the immune system, mediating the growth and metastasis of tumours, and negatively influences the prognosis of cancer patients. The AGR has been recognized as a classic factor for the prediction of prognosis in renal cancer. $^{34,35}$ In this study, our results have verified that 
A

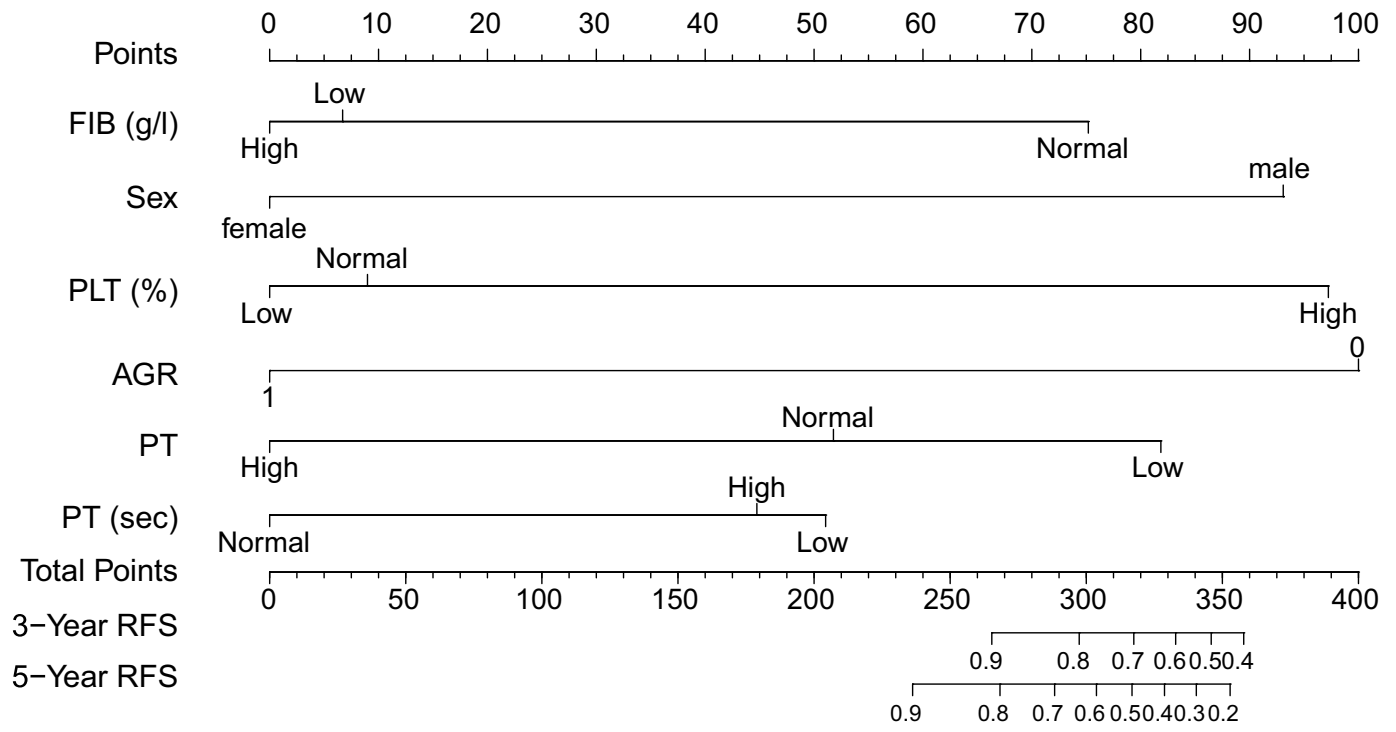

B

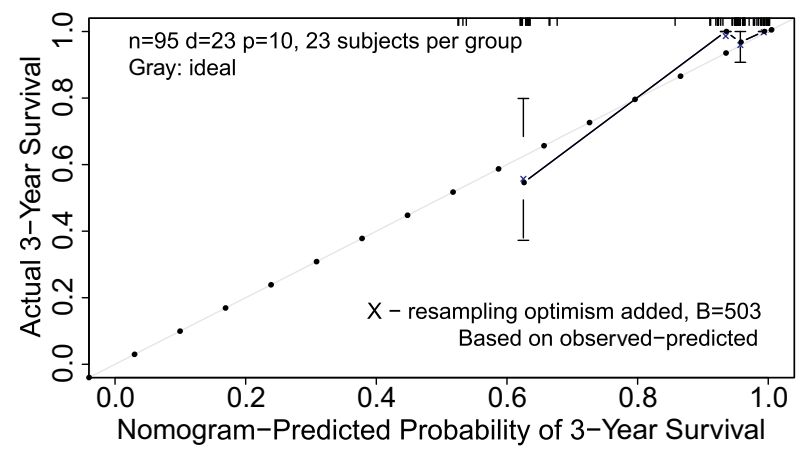

D

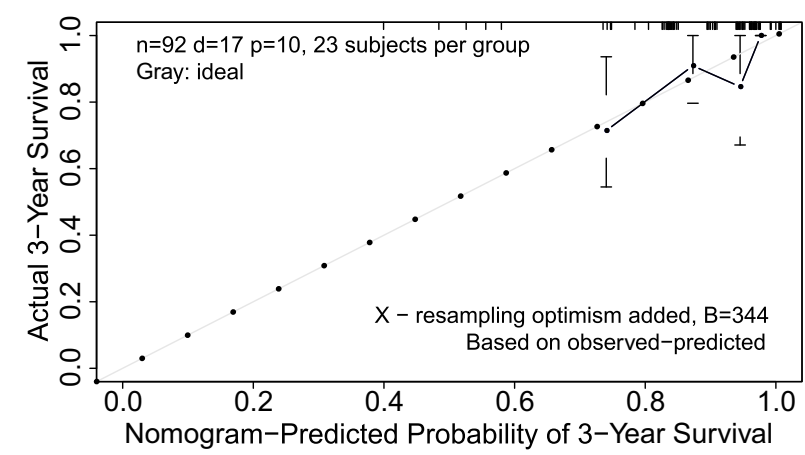

C

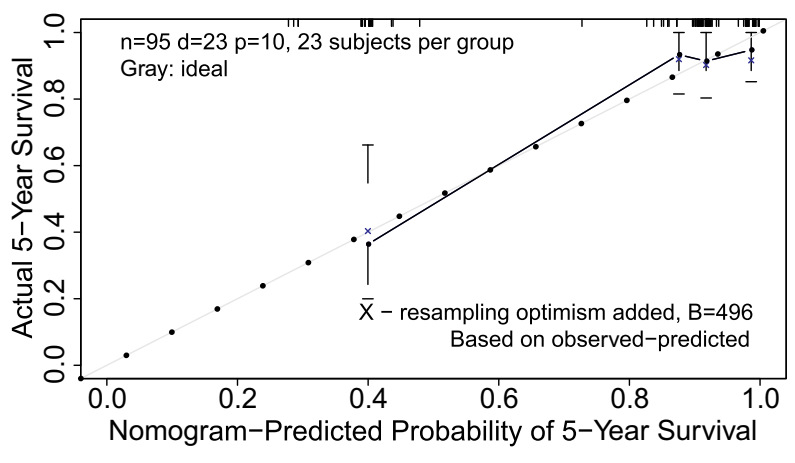

E

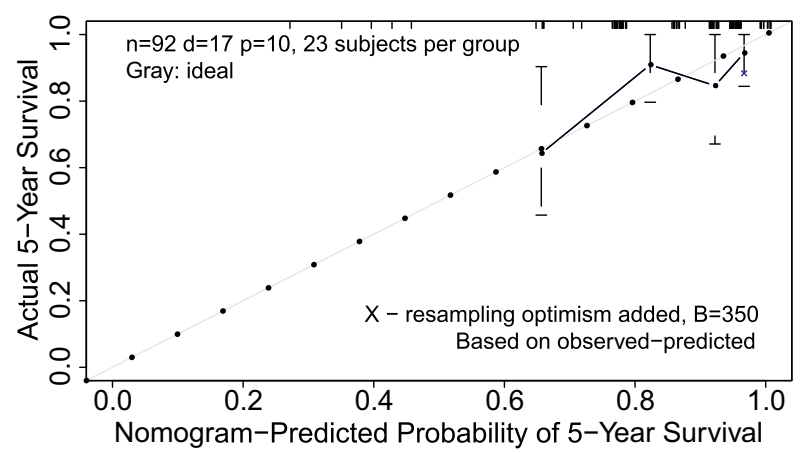

Figure 2 Construction of a nomogram for recurrence-free survival predicting. (A) Nomogram combining signature with laboratory results and clinicopathological features. (B-E) Calibration plot displaying that the nomogram model predicted recurrence-free survival probabilities was proved consistent with the actual observed proportions.

a low AGR may represent the poor prognosis of patients with renal cancer.

Elevated blood platelets could be considered a potential risk factor in the development of kidney cancer. Thrombocytosis is most likely associated with the neuroendocrine activity of tumour cells. There is evidence of the protective effect of platelets in relation to circulating tumour cells. They escaped the regulation of the immune system by hiding their recognition and facilitating their integration into the endothelium. ${ }^{21}$ Platelets potentially influence tumour growth by generating growth factors, such as PDGF and VEGF. Studies have indicated that the renal cancer patients who have thrombocytosis mostly have unfavourable prognoses after surgery. ${ }^{21,30,36}$ Our 
A

Training set

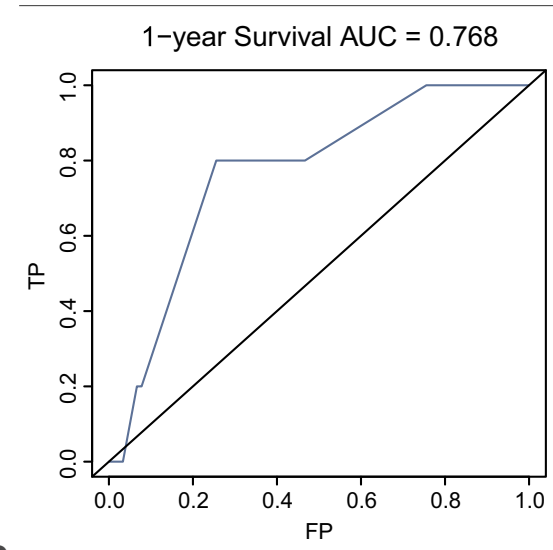

B

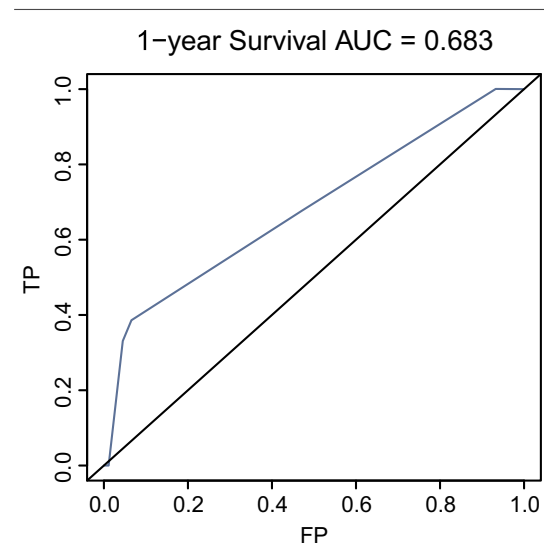

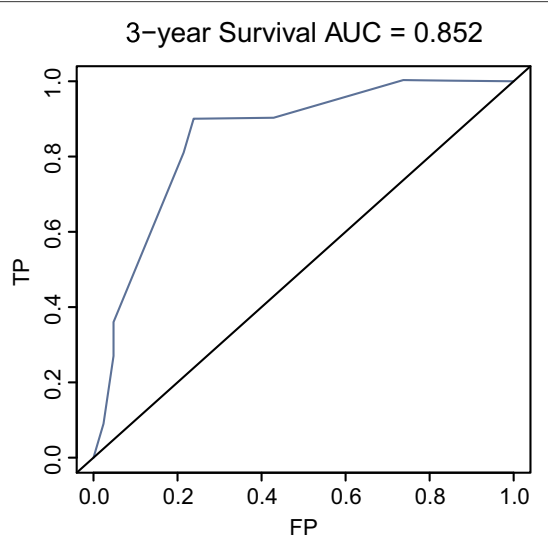

Validation set
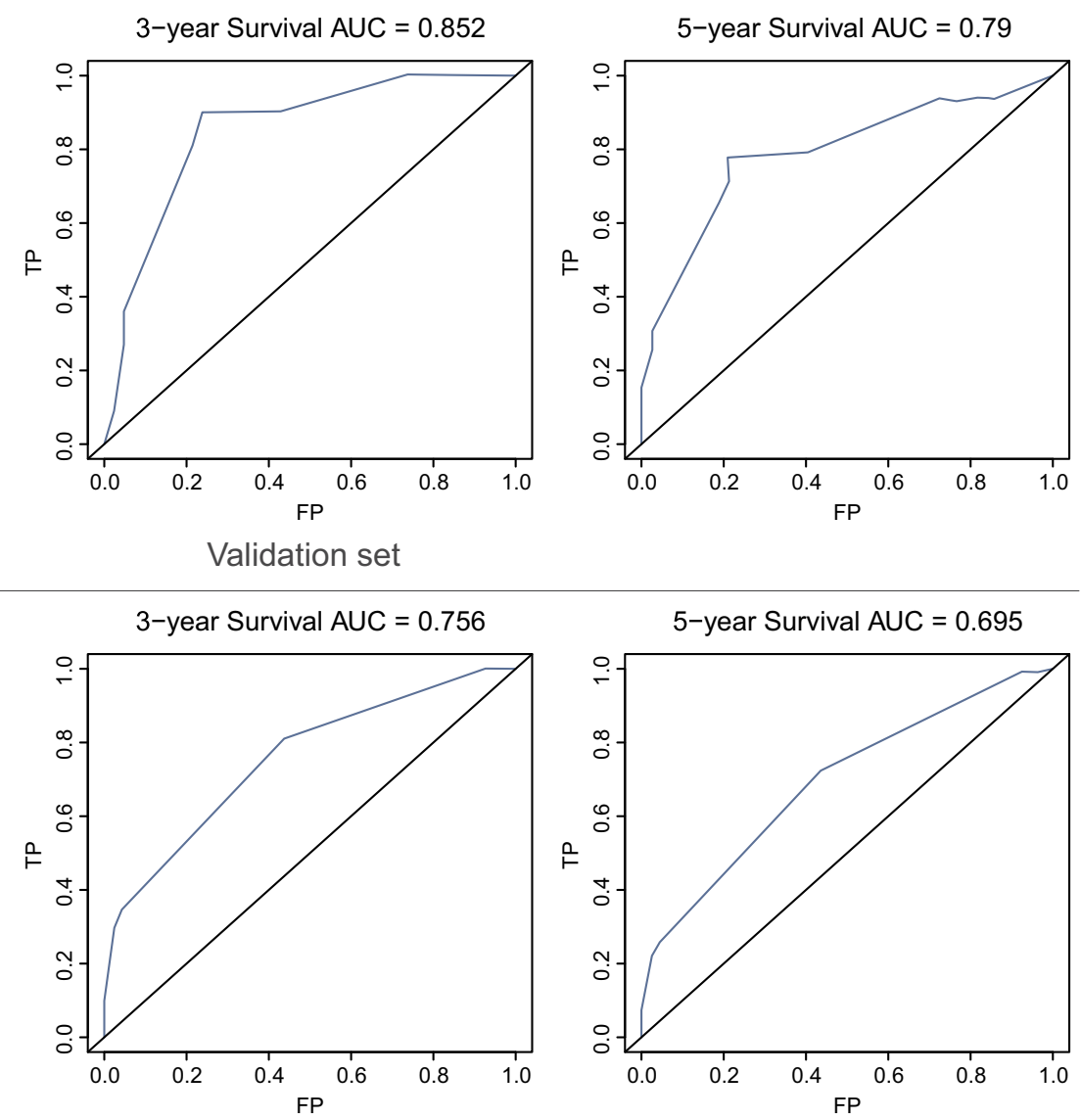

Figure 3 ROC curve analyses for the nomogram in predicting recurrence-free survival. (A) ROC curves showed satisfied predictive values of the nomogram in the training cohort at I-, 3- and 5-year. (B) ROC curves showed satisfied predictive values of the nomogram in the validation cohort at I-, 3- and 5-year.

Abbreviation: ROC, receiver operating characteristic.

A

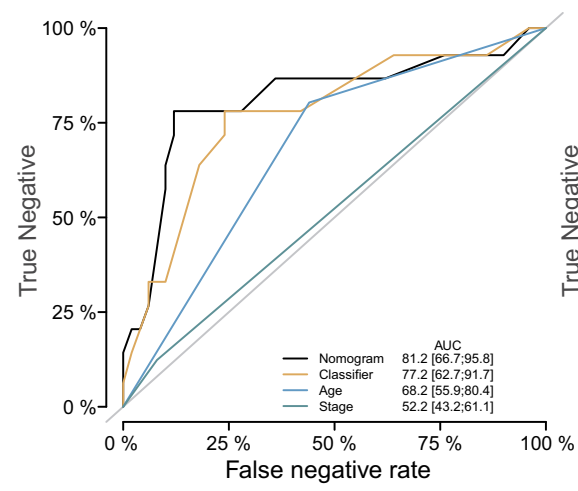

B

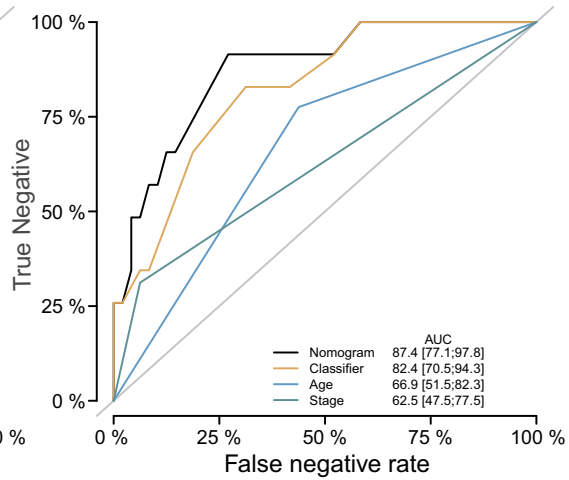

C

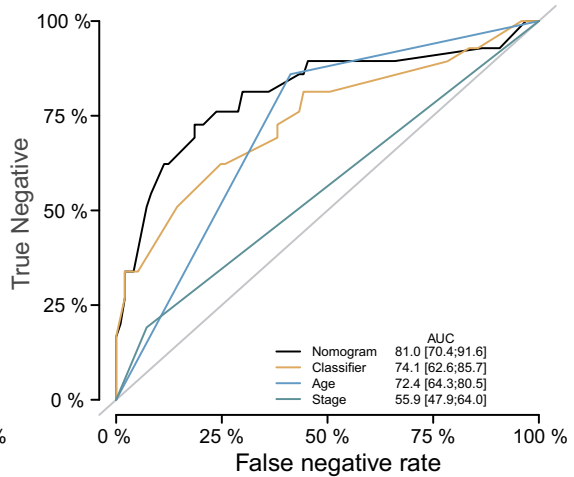

Figure 4 Nomogram (version 2) showed increased predictive values by combining the six-variables-based classifier and clinicopathological features in the training (A), validation (B) and overall datasets (C).

study has indicated that a high level of PLT could be a risk factor for the recurrence of renal cancer.

As the incidence of most cancers increases along with the age, cancer is considered an age-associated disease and begins to rise faster in middle age. Some biological mechanisms which regulate ageing might also be involved in the pathogenesis of age-related diseases, such as cancer. In addition, gender has also been proven to be associated with the 
A
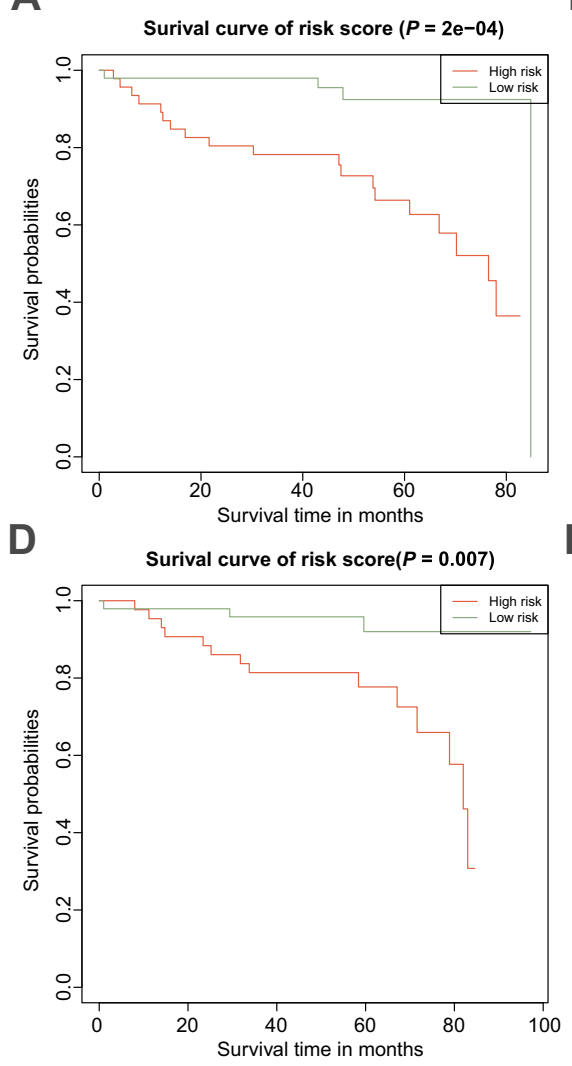

B

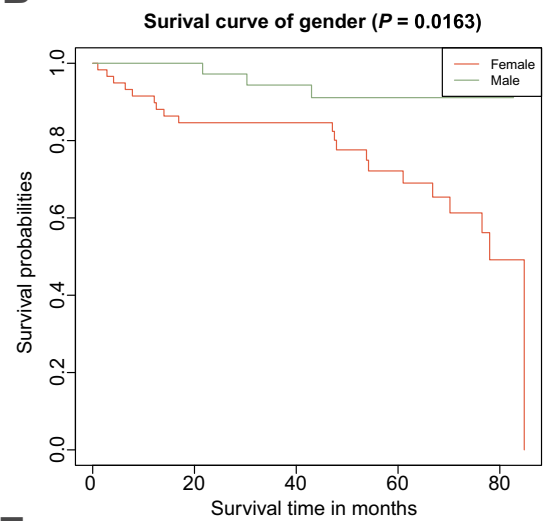

$\mathrm{E}$

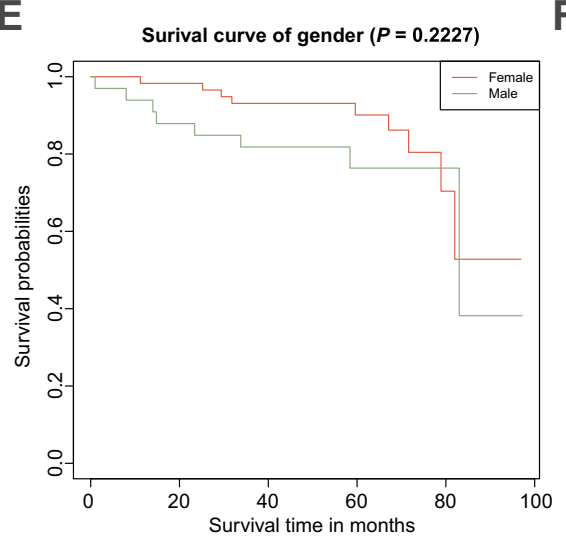

C

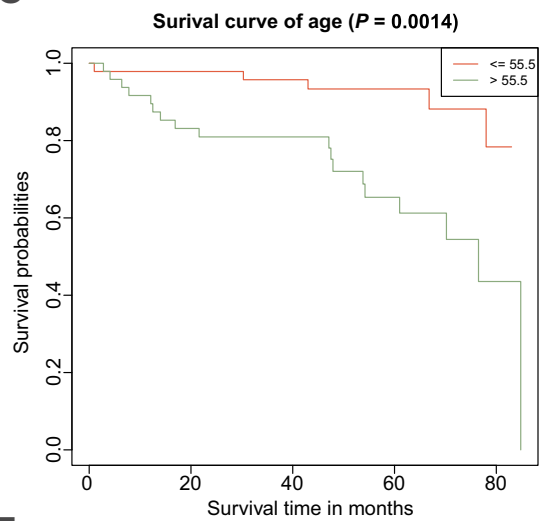

$\mathbf{F}$

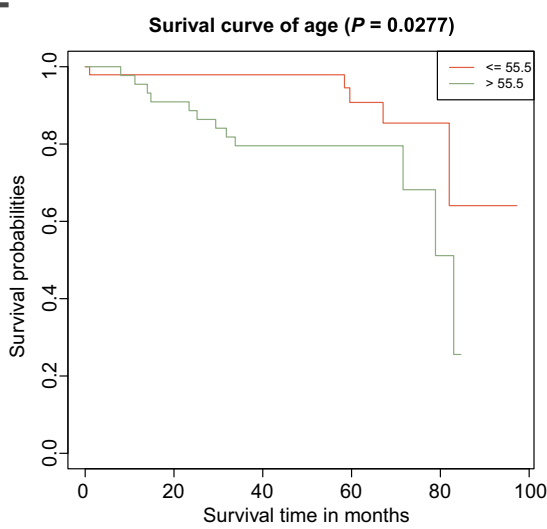

Figure 5 Survival analyses of the nomogram in predicting recurrence-free survival of renal cancer patients. (A-C) Stratified survival analyses based on the risk score, gender and age in the training cohort. (D-F) Stratified survival analyses based on the risk score, gender and age in the validation cohort.

prognosis of renal cancer patients. In our study, we found female patients had a better RFS status than male patients. $\mathrm{Qu}$ et $\mathrm{al}^{37}$ suggested that, for these young and premenopausal women, they mostly had good survival status, which might be conferred by the oestrogen axis. In addition, the protective effect of the oestrogen axis on prognosis has also been observed in other cancers, such as colorectal cancer. ${ }^{38}$ Previous basic research found that the oestrogen-ER $\beta$ axis inhibits the proliferation and induces the apoptosis of renal cancer cells. ${ }^{38}$ Thus, increased circulating levels of oestrogen may enhance the tumour suppressor function of $E R b$, improving the prognosis of female patients with renal cancer.

Advantages are obvious for the current study. We defined a novel method to obtain a more accurate prediction of the recurrence of renal cancer after surgery. All the enrolled factors, including the results of blood coagulation factors, are normally tested and recorded for the inpatient patients, so there is no extra economic burden. However, we could use these results to get a better prediction of the prognosis for renal cancer patients. Better prediction means better preparation for the precise treatment. Limitations of this study exist in the cohort design and its retrospective nature, thereby allowing intrinsic biases that may affect the results. Patients with renal cancer are a heterogeneous group, from which we have a relatively small sample size and a diverse range of histology. Our findings that rely on the pathological features and laboratory test results require external validation. Currently, few established prognostic models are completely foolproof, even if they have enough ability to predict prognosis. In this case, more accurate markers are continually being searched for. The different biologic behaviours underlying the different clinical scenarios unveiled by molecular events may help make the risk-stratified clinical decision and provide personal prognostication. ${ }^{39}$ In the targeted therapies epoch, localized and metastatic renal cancer patients demand the latest models and new prognostic factors. ${ }^{40}$

In conclusion, the nomogram, as proposed in the current study, objectively and accurately predicts the RFS of renal cancer patients after surgery. Multicentre prospective studies are warranted to validate our findings. 


\section{Data Sharing Statement}

The anonymized data used and/or analysed during the current study are available from the corresponding authors on reasonable request.

\section{Ethics Approval and Consent to Participate}

The contents and methods of the research were reviewed and approved by the Ethics Committee of the First Affiliated Hospital of Anhui Medical University (anyiyifuyuanlunshen-kuai-PJ-2019-09-11). This study only collecting limited data that is known only to researchers, and the results of the research will not affect clinical care of the individuals, since they will already have left the hospital. Therefore, the consent to review the medical records were not required by the Ethics Committee of the First Affiliated Hospital of Anhui Medical University. Patient information and clinical records were anonymized and deidentified prior to analysis. The study was conducted in compliance with the Declaration of Helsinki principles.

\section{Consent for Publication}

All authors have read and approved the manuscript being submitted and published, and agree to its submitted journal.

\section{Acknowledgments}

This study was supported by The National Natural Science Foundation of China 81802827, 81630019 and 81870519, Scientific Research Foundation of the Institute for Translational Medicine of Anhui Province (2017ZHYX02), The Natural Science Foundation of Guangdong Province, China (2017A030313800), and Shenzhen Basic Research Project (JCYJ20160429172247015).

\section{Author Contributions}

All authors made a significant contribution to the work reported, whether that is in the conception, study design, execution, acquisition of data, analysis and interpretation, or in all these areas; took part in drafting, revising or critically reviewing the article; gave final approval of the version to be published; have agreed on the journal to which the article has been submitted; and agree to be accountable for all aspects of the work.

\section{Disclosure}

All the authors state they have no conflicts of interest.

\section{References}

1. Siegel RL, Miller KD, Jemal A. Cancer statistics, 2018. CA Cancer J Clin. 2018;68(1):7-30. doi:10.3322/caac.21442

2. Psutka SP, Master VA. Role of metastasis-directed treatment in kidney cancer. Cancer. 2018;124(18):3641-3655. doi:10.1002/cncr.31341

3. Tunio MA, Hashmi A, Rafi M. Need for a new trial to evaluate postoperative radiotherapy in renal cell carcinoma: a meta-analysis of randomized controlled trials. Ann Oncol. 2010;21(9):1839-1845. doi:10.1093/annonc/mdq028

4. Shen WF, Zhong W, Xu F, et al. Clinicopathological and prognostic analysis of 429 patients with intrahepatic cholangiocarcinoma. World J Gastroenterol. 2009;15(47):5976-5982. doi:10.3748/wjg.15.5976

5. Zhang S, Huang X, Tian Y, et al. Clinicopathologic characteristics, laboratory parameters, treatment protocols, and outcomes of pancreatic cancer: a retrospective cohort study of 1433 patients in China. PeerJ. 2018;6:e4893. doi:10.7717/peerj.4893

6. Wang B, Yang Y, Jiang Z, et al. Clinicopathological characteristics, diagnosis, and prognosis of pregnancy-associated breast cancer. Thorac Cancer. 2019;10(5):1060-1068. doi:10.1111/1759-7714.13045

7. Xie Y, Ma X, Li H, et al. Prognostic value of clinical and pathological features in chinese patients with chromophobe renal cell carcinoma: a 10-year single-center study. $J$ Cancer. 2017;8(17):3474-3479. doi:10.7150/jca. 19953

8. Gupta D, Lis CG. Pretreatment serum albumin as a predictor of cancer survival: a systematic review of the epidemiological literature. Nutr J. 2010;9:69. doi:10.1186/1475-2891-9-69

9. Fidler MM, Bray F. Global cancer inequalities. Front Oncol. 2018;8:293. doi:10.3389/fonc.2018.00293

10. Zhang K, Gao HF, Mo M, et al. A novel scoring system based on hemostatic parameters predicts the prognosis of patients with advanced pancreatic cancer. Pancreatology. 2019;19(2):346-351. doi:10.1016/j.pan.2018.12.010

11. Wang JM, Wang Y, Huang YQ, et al. Prognostic values of platelet-associated indicators in resectable cervical cancer. Dose Response. 2019;17(3):1559325819874199.

12. Wang RT, Zhang LQ, Mu YP, et al. Prognostic significance of preoperative platelet count in patients with gallbladder cancer. World $J$ Gastroenterol. 2015;21(17):5303-5310. doi:10.3748/wjg.v21. i17.5303

13. Lima LG, Monteiro RQ. Activation of blood coagulation in cancer: implications for tumour progression. Biosci Rep. 2013;33:5. doi:10.1042/BSR20130057

14. Ferrigno D, Buccheri G, Ricca I. Prognostic significance of blood coagulation tests in lung cancer. Eur Respir J. 2001;17(4):667-673. doi:10.1183/09031936.01.17406670

15. Nakamura K, Nakayama K, Ishikawa $M$, et al. High pretreatment plasma D-dimer levels are related to shorter overall survival in endometrial carcinoma. Eur J Obstet Gynecol Reprod Biol. 2016;201:89-93. doi:10.1016/j.ejogrb.2016.03.041

16. Li Q, Cong R, Kong F, Ma J, Wu Q, Ma X. Fibrinogen is a coagulation marker associated with the prognosis of endometrial cancer. Onco Targets Ther. 2019;12:9947-9956. doi:10.2147/OTT. S222813

17. Gupta K, Miller JD, Li JZ, Russell MW, Charbonneau C. Epidemiologic and socioeconomic burden of metastatic renal cell carcinoma (mRCC): a literature review. Cancer Treat Rev. 2008;34 (3):193-205. doi:10.1016/j.ctrv.2007.12.001

18. Chen K, Lee BL, Huang HH, et al. Tumor size and Fuhrman grade further enhance the prognostic impact of perinephric fat invasion and renal vein extension in T3a staging of renal cell carcinoma. Int J Urol. 2017;24(1):51-58. doi:10.1111/iju.13237

19. Wu CY, Huo JP, Zhang XK, et al. Loss of CD15 expression in clear cell renal cell carcinoma is correlated with worse prognosis in Chinese patients. Jpn J Clin Oncol. 2017;47(12):1182-1188. doi:10.1093/jjco/hyx139 
20. Sternberg CN. Are nomograms better than currently available stage groupings for bladder cancer? J Clin Oncol. 2006;24(24):3819-3820. doi:10.1200/JCO.2006.07.1290

21. Touijer K, Scardino PT. Nomograms for staging, prognosis, and predicting treatment outcomes. Cancer. 2009;115(13 Suppl):3107-3111. doi:10.1002/cncr.24352

22. Amirkhosravi A, Meyer T, Amaya M, et al. The role of tissue factor pathway inhibitor in tumor growth and metastasis. Semin Thromb Hemost. 2007;33(7):643-652. doi:10.1055/s-2007-991531

23. Falanga A, Marchetti M, Vignoli A, Balducci D. Clotting mechanisms and cancer: implications in thrombus formation and tumor progression. Clin Adv Hematol Oncol. 2003;1(11):673-678.

24. Langer F. Haemostatic aspects in clinical oncology. Hamostaseologie. 2015;35(2):152-164; quiz 165. doi:10.5482/ HAMO-14-11-0057

25. McAlister RK, Ito S. Minimal prolongation of prothrombin time with extended exposure to argatroban. Pharmacotherapy. 2015;35(7): e122-e126. doi:10.1002/phar.1613

26. Li XH, Wang XP, Gu WS, et al. Clinical significance of preoperative thrombin time in patients with esophageal squamous cell carcinoma following surgical resection. PLoS One. 2015;10(10): 0140323. doi:10.1371/journal.pone.0140323

27. Tas F, Kilic L, Serilmez M, Keskin S, Sen F, Duranyildiz D. Clinical and prognostic significance of coagulation assays in lung cancer. Respir Med. 2013;107(3):451-457. doi:10.1016/j.rmed.2012.11.007

28. Bottasso B, Mari D, Coppola R, Santoro N, Vaglini M, Mannucci PM. Hypercoagulability and hyperfibrinolysis in patients with melanoma. Thromb Res. 1996;81(3):345-352. doi:10.1016/ 0049-3848(96)00006-0

29. Shu YJ, Weng H, Bao RF, et al. Clinical and prognostic significance of preoperative plasma hyperfibrinogenemia in gallbladder cancer patients following surgical resection: a retrospective and in vitro study. BMC Cancer. 2014;14:566. doi:10.1186/1471-2407-14-566

30. Ramsey S, Lamb GW, Aitchison M, McMillan DC. Prospective study of the relationship between the systemic inflammatory response, prognostic scoring systems and relapse-free and cancer-specific survival in patients undergoing potentially curative resection for renal cancer. BJU Int. 2008;101(8):959-963. doi:10.1111/j.1464-410X. 2007.07363.x
31. Mei Y, Liu H, Sun X, Li X, Zhao S, Ma R. Plasma fibrinogen level may be a possible marker for the clinical response and prognosis of patients with breast cancer receiving neoadjuvant chemotherapy. Tumour Biol. 2017;39(6):1010428317700002. doi:10.1177/ 1010428317700002

32. Gabay C, Kushner I, Epstein FH. Acute-phase proteins and other systemic responses to inflammation. $N$ Engl J Med. 1999;340 (6):448-454. doi:10.1056/NEJM199902113400607

33. McMillan DC, Watson WS, O'Gorman P, Preston T, Scott HR, McArdle CS. Albumin concentrations are primarily determined by the body cell mass and the systemic inflammatory response in cancer patients with weight loss. Nutr Cancer. 2001;39(2):210-213. doi:10.1207/S15327914nc392_8

34. Chen Z, Shao Y, Yao H, et al. Preoperative albumin to globulin ratio predicts survival in clear cell renal cell carcinoma patients. Oncotarget. 2017;8(29):48291-48302. doi:10.18632/ oncotarget.15162

35. Koparal MY, Polat F, Cetin S, Bulut EC, Sozen TS. Prognostic role of preoperative albumin to globulin ratio in predicting survival of clear cell renal cell carcinoma. Int Braz J Urol. 2018;44(5):933-946. doi:10.1590/s1677-5538.ibju.2018.0012

36. Prokopowicz G, Zyczkowski M, Nowakowski K, Bogacki R, Bryniarski P, Paradysz A. Basic parameters of blood count as prognostic factors for renal cell carcinoma. Biomed Res Int. 2016;2016:8687575. doi:10.1155/2016/8687575

37. Qu Y, Chen H, Gu W, et al. Age-dependent association between sex and renal cell carcinoma mortality: a population-based analysis. Sci Rep. 2015;5:9160. doi:10.1038/srep09160

38. Yu CP, Ho JY, Huang YT, et al. Estrogen inhibits renal cell carcinoma cell progression through estrogen receptor- $\beta$ activation. PLoS One. 2013;8(2):e56667. doi:10.1371/journal.pone.0056667

39. Sun M, Shariat SF, Cheng C, et al. Prognostic factors and predictive models in renal cell carcinoma: a contemporary review. Eur Urol. 2011;60(4):644-661. doi:10.1016/j.eururo.2011.06.041

40. Sun M, Shariat SF, Karakiewicz PI. Factors affecting outcome in renal cell carcinoma. Curr Opin Urol. 2010;20(5):355-360. doi:10.1097/MOU.0b013e32833c7b19

\section{Publish your work in this journal}

Cancer Management and Research is an international, peer-reviewed open access journal focusing on cancer research and the optimal use of preventative and integrated treatment interventions to achieve improved outcomes, enhanced survival and quality of life for the cancer patient.
The manuscript management system is completely online and includes a very quick and fair peer-review system, which is all easy to use. Visit http://www.dovepress.com/testimonials.php to read real quotes from published authors. 